\title{
Correction to: Uncertainty Assessment over any Volume without Simulation: Revisiting Multi-Gaussian Kriging
}

\author{
Álvaro I. Riquelme ${ }^{1}\left[\right.$ IC $\cdot$ Julian M. Ortiz $^{1}$
}

Published online: 25 February 2021

(C) International Association for Mathematical Geosciences 2021

\section{Correction to: Math Geosci https://doi.org/10.1007/s11004-020-09907-9}

The publisher apologized for this unfortunate error, which has been corrected in the original article. Due to a processing error, all upper sigma symbols, denoting covariance matrices, appeared without bold. All upper sigma Greek letters throughout the paper should be in bold. For example, in page 4

$$
f_{Y_{1}, \ldots, Y_{N}}\left(y_{1}, \ldots, y_{N}\right)=\frac{\exp \left(-\frac{1}{2}(\boldsymbol{y}-\boldsymbol{\mu})^{T} \boldsymbol{\Sigma}^{-1}(\boldsymbol{y}-\boldsymbol{\mu})\right)}{\sqrt{(2 \pi)^{N}|\boldsymbol{\Sigma}|}},
$$

In addition, an error was inserted at the end of page 6, wherein the symbols $\mu$ and $\sigma$ where replaced by words. Please read this last paragraph as

"We define as $G_{\mu}^{\sigma^{2}}$ the cdf of a Gaussian RF with mean $\mu$ and variance $\sigma^{2}$, except for the standard case, noted as $G$. The notation for the Gaussian probability density function (pdf) with mean $\mu$ and variance $\sigma^{2}$ is introduced as $g_{\mu}^{\sigma^{2}}$, except for the standard case, noted as $g$. Then, Eq. (2) can be written as $F_{Z(\mathbf{u})} \circ \phi=G$."

The original article can be found online at https://doi.org/10.1007/s11004-020-09907-9.

Álvaro I. Riquelme

alvaro.riquelme@queensu.ca

Julian M. Ortiz

julian.ortiz@queensu.ca

1 Robert M. Buchan Department of Mining, Queen's University, Kingston, Canada 
In addition, an error was inserted in the equation at the middle of page 9, where the symbols for dots where replaced by words. Please read this equation as

$$
\begin{aligned}
F_{Z\left(\mathbf{u}_{1}\right), \ldots, Z\left(\mathbf{u}_{N}\right) \mid \text { data }}\left(z_{1}, \ldots, z_{N}\right) & =\mathbb{P}\left(Z\left(\mathbf{u}_{1}\right) \leq z_{1}, \ldots, Z\left(\mathbf{u}_{N}\right) \leq z_{N} \mid \text { data }\right) \\
& =\mathbb{P}\left(Y\left(\mathbf{u}_{1}\right) \leq y_{1}, \ldots, Y\left(\mathbf{u}_{N}\right) \leq y_{N} \mid \text { data }\right) \\
& =F_{Y\left(\mathbf{u}_{1}\right), \ldots, Y\left(\mathbf{u}_{N}\right) \mid \text { data }}\left(y_{1}, \ldots, y_{N}\right) \\
& =G_{\mu_{S K}}^{\Sigma_{S K}}\left(y_{1}, \ldots, y_{N}\right) .
\end{aligned}
$$

In addition, an error was inserted at the end of page 15. Please read as $\sigma_{S K}^{2}\left(\mathbf{u}_{i}\right)$ instead of $\sigma_{S}^{2} K\left(\mathbf{u}_{i}\right)$. 